\title{
Requirements for biosimilar authorization: a legal and comparative perspective - FDA versus EMA
}

\author{
Alfonso Noguera Peña* and Carlos del Castillo Rodríguez
}

Both European Medicines Agency (EMA) and Food and Drug Administration (FDA) have developed a regulatory framework for the authorization of biosimilar medicines. Marketing authorization application must demonstrate that the biosimilar proposed has similar physicochemical, biological, clinical and non-clinical profiles to a reference biological medicine, which could be marketed upon expiration of the patent and data protection period of the innovative medicine. Although the basic principles to demonstrate biosimilarity are equivalent, there are significant differences in certain aspects. The biosimilarity criteria have not remained static, observing a reduction in the regulatory requirements established by EMA. On the other hand, several proposals concerning the amendment of biosimilarity demonstration have been suggested.

Keywords: Authorization, biosimilar medicines, legal perspective, regulatory framework.

\section{Biosimilar medicine}

BIOSIMILAR medicines represent one of the several variants known as biopharmaceuticals. According to the definition proposed by the European Medicines Agency (EMA), a biosimilar 'is a product which is similar to a biological medicine that has already been authorized" contains a known biological active substance, which is similar to that of the reference medicine.

According to the definition of the Food and Drug Administration (FDA), USA, a biosimilar: 'is a biological product highly similar to the reference product notwithstanding minor differences in clinically inactive components'. Furthermore, it adds that there are 'no clinically meaningful differences between the biologic product and the reference product in terms of the safety, purity, and potency of the product ${ }^{2}$.

The main difference between the two definitions lies in the terminology. While the EMA requires similarity between the reference and candidate medicines as

\footnotetext{
Alfonso Noguera Peña is in the Pharmacy Military Centre of Defence. Logistic Base San Pedro, Highway M-609 Miraflores, km 34, E-28770, Colmenar Viejo (Madrid), Spain; Universidad Complutense de Madrid, History of Pharmacy and Pharmaceutical Legislation, Department of Galenic Pharmacy and Food Technology. Ramon y Cajal Square s/n, E28040, Madrid (Madrid), Spain; Carlos del Castillo Rodríguez is in the Universidad Complutense de Madrid, History of Pharmacy and Pharmaceutical Legislation, Department of Galenic Pharmacy and Food Technology. Ramon y Cajal Square s/n, E-28040, Madrid (Madrid), Spain.*For correspondence. (e-mail: alfnogue@ucm.es)
}

biosimilar in terms of 'quality, efficacy, and safety', the FDA refers to 'safety, purity, and potency'.

Biosimilar medicines present the same mechanism of action as the reference medicine. They also present highly similar structural, functional and physico-chemical characteristics with regard to the latter. In addition, the toxicity, pharmacokinetic (PK) and pharmacodynamic (PD) profiles are comparable. It is expected that biosimilar medicines have a similar quality, efficacy and safety with respect to the innovative medicine ${ }^{3}$.

The biosimilarity relationship between two given medicines is not transitive ${ }^{4}$, that is, the authorized medicine as a biosimilar has only demonstrated this relationship with the reference medicine, and not with the rest of the biosimilar medicines.

\section{Concepts related to biosimilar medicines}

Biosimilar medicines are authorized in certain territories that have adopted a consolidated regulatory framework to standardize them. In addition, the regulatory agencies responsible for evaluation of these medicines have adopted a decision on marketing and subsequent marketing authorization through the study of the differences between the reference medicine and the biosimilar medicine candidate by means of an extensive and rigorous comparative exercise ${ }^{5}$. Thus, in the regions where more relaxed requirements have been adopted for the marketing of biological medicines that are similar to a reference medicine, the following terms, among others, have been coined: 
'non-original biologics', 'non-comparable biologics' or 'intended copies'.

However, the last few years have witnessed a strengthening of the regulation regarding biosimilar medicines in countries outside the International Council for Harmonisation of Technical Requirements for Pharmaceuticals for Human Use (ICH), like India, for example ${ }^{6,7}$. Therefore, we consider that probably in the near future these terms would become obsolete, due to a consensus in the basic regulatory requirements for the authorization of biosimilar medicines at a global level.

However, the EMA and FDA do not allow that the medicine, for which a biosimilar status is claimed, improves the guarantees of efficacy with respect to the innovative medicine. In these cases, the medicine should be authorized by a not abbreviated procedure, as any other original biological medicine. These medicines are referred to as 'biobetter' or 'biosuperior', although the regulatory agencies currently do not recognize them officially.

Other medicines related with these are the so-called 'stand-alone ${ }^{, 8,9}$. These medicines could probably be authorized through the demonstration of their similarity with a reference biological medicine. However, if the applicant decides to carry out a full development, they would be authorized as any other original medicines.

On the other hand, biosimilar medicines are not generic or biogeneric ${ }^{10}$. Due to the inherent variability of living beings and production processes of biological medicines, as well as to the availability of analytical techniques of high sensibility, there are physico-chemical differences between the innovative medicine and its biosimilars that can be observed, as for example, the extent of glycosylation, deamination, methylation, loading variants, etc. ${ }^{4,5,11,12}$. However, the differences detected between the two products should not represent a significant impact regarding quality, safety and/or efficacy. These differences will be evaluated by the competent regulatory authorities. For these reasons, it is easy to note that for biological medicines, 'the product is the process', and that biosimilar medicines are 'similar, but not identical' with respect to the innovative or reference medicine.

\section{Advantages and disadvantages of the marketing of biosimilar medicines}

Biosimilar medicines present several benefits to all stakeholders $^{13-16}$. For the pharmaceutical industry, they represent an advance in the research and development field. For patients, they are an additional therapeutic alternative, they improve the administration devices, increase the therapeutic adherence and enhance the accessibility to biological treatments, as they facilitate the treatment of naïve patients ${ }^{17}$. For health authorities, they mean savings in pharmaceutical expenses, as biosimilar medicines are marketed at a lower price compared to the reference medicines (the percentage of reduction is about $15-40 \%)^{18}$. Besides, by increasing competition in the market $^{19}$, they also induce a reduction in the price of innovative medicines.

Therefore, due to savings in public pharmaceutical spending, biosimilar medicines facilitate the sustainability of public health systems, and also facilitate the accessibility of treatments to patients; hence their increased social interest $\mathrm{t}^{20}$. In Spain, savings between 2009 and 2016 have been estimated as not less than 478 million euros ${ }^{21}$.

Given that a large part of the world's population does not have sufficient financial resources at its disposal to pay for the most basic medical treatments, it will be difficult for people to access essential medicines with high economic impact (including biological medicines); hence the health and social interests of the commercialization of biosimilar medicines.

However, the marketing of biosimilar medicines has not been free from controversies, especially regarding extrapolation of indications ${ }^{22}$, immunogenicity ${ }^{23}$, pharmacovigilance ${ }^{24}$, substitution ${ }^{25}$ and interchangeability $3,20,26-29$.

In this study we analyse the concept of biosimilarity as well as the regulatory requirements for the authorization of biosimilar medicines of the EMA and FDA, as they are the main regulatory territories where a solid framework has been adopted for the marketing of biosimilar medicines.

\section{Legal instruments that limit the marketing of biosimilar medicines}

\section{European Union}

Biosimilar medicines are developed once the exclusive industrial exploitation rights of the original, innovative or reference medicine have expired, or, in other words, once the patent of the reference medicine expires. Therefore, biosimilar medicines could be marketed in the European Union (EU) 20 years after the inscription of the original medicine patent.

To register a biosimilar medicine, protection of reference medicine by legal instruments of commercial nature (for example, patents) is not the only factor that is considered, but also exclusivity period of the data provided by the competent regulatory authorities.

In the EU, through Directive 2004/27/EC of the European Parliament and of the Council, of 31 March 2004, amending Directive 2001/83/EC, a single period of exclusivity of the data, fixed in 10 years, was established for innovative medicines and harmonized throughout Europe. Furthermore, the said period will be extended up to a maximum of 11 years if, during the first eight of the ten-year period, the laboratory that holds the marketing authorization of the reference medicine obtains authorization for one or several new therapeutic indications, and if, 
during prior scientific evaluation, it is established that those indications would provide a significant clinical benefit in comparison with the existing therapies. This data protection period is known as 'Bolar provision'.

\section{United States of America}

In USA, by reason of section 351(k) of the Biologics Price Competition and Innovation Act (BPCI Act) of 2009 , a single exclusivity period of the data of 12 years is established for the reference biological medicine. Nevertheless, the mentioned legal provision prevents requests for the authorization of a biosimilar medicine by any pharmaceutical laboratory until four years after the health registration of the reference medicine.

In addition, regardless of the rights granted by the patent, as well as the protection period of the industrial data, in the case of biosimilar medicines, if the applicant demonstrates that it is interchangeable with the reference medicine, then the FDA grants a year of commercial exclusivity for the first biosimilar marketed with a certain active substance.

\section{Requirements for the marketing of biosimilar medicines}

The pharmaceutical laboratories interested in obtaining a health authorization for the marketing of biosimilar medicines must provide the competent authorities with a series of comparative studies to demonstrate the similarity of the medicine proposed, as a biosimilar with respect to the innovative medicine in terms of quality, safety and efficacy ${ }^{8,30,31}$. These characteristics, along with information and identification, are the basic requirements that are demandable in order to market the medicines in the EU and USA.

Ultimately, biosimilar medicines are authorized through a specific and accelerated procedure, as the health authorities do not demand to perform a full pre-clinical and clinical development, as is the case of reference medicines or 'biobetters'. This is so because the reference medicine has already been demonstrated as safe and efficient for the authorized therapeutic indications. The biosimilar medicine must demonstrate to be comparable with the reference medicine, as well as presenting a favourable benefit/risk balance.

Due to the special characteristics of biological medicines, direct application of the regulatory law of generic medicines would not be consistent; hence the need to develop a specific regulatory framework for biosimilar medicines. However, from the legal health perspective we understand that such a fact generates legal insecurity, a specific regulation for biosimilar medicines is needed, in order to protect the rights of patients to access the appropriate biological medicine.
In general, both the EMA and FDA have adopted soft law dispositions (guidance, guidelines), that have a recommendation or suggestion. Therefore, unlike other types of regulatory dispositions (Directive, Regulation), they are not mandatory and also do not generate binding legal effects for third parties (Table 1).

The EMA has been the pioneer in the development of a regulatory framework for the authorization of biosimilar medicines. The first version of the guideline on similar biological medicinal products entered into force in 2005 (ref. 30). A year later, the first biosimilar medicine was authorized in the EU. To date, more than 60 biosimilar medicines have been authorized within the EMA. Thus, there is a consolidated regulatory framework, as well as a significant experience with their use. However, in USA, biosimilar medicines began to be marketed in 2015. So, experience in the use of these medicines is lower in USA compared to EU.

\section{Requirements for reference medicine}

The innovative or reference medicine must have been authorized in the regulatory territory (in the European Economic Area (EEA), or USA) where the biosimilar medicine is intended to be marketed, through a complete Common Technical Document ${ }^{8,31}$. In other words, a reference medicine that has not been authorized in the regulatory territory where a marketing authorization is intended to be obtained cannot be chosen; and the use of a biosimilar medicine that has been previously authorized in the same region as a reference medicine, is also not allowed.

Since one of the cornerstones in the development of biosimilar medicines is based on the application of the $3 R$ rule (replacement, reduction, refinement) ${ }^{32}$, it is clear that we need to avoid the redundant duplication of clinical trials. Not only because this would be wrong from the ethical point of view, but because it would hinder the global development of these medicines. Thus, both the EMA (from October 2014 onwards) and the FDA, allow the applicant to compare a given biosimilar medicine in certain clinical trials and non-clinical in vivo studies with a comparator that is not authorized in the regulatory territory where the authorization application of the biosimilar medicine has been processed. That non-authorized comparator must be an innovative medicine authorized by a medicine regulatory authority included in the $\mathrm{ICH}$, or that maintains similar scientific and regulatory standards for the marketing of biosimilar medicines.

If the applicant chooses to use a non-authorized comparator in some studies leading to the demonstration of biosimilarity, he/she must demonstrate that the nonauthorized comparator represents the reference medicine authorized by the EMA/FDA (as appropriate). It would also be necessary to provide an adequate scientific 
GENERAL ARTICLES

Table 1. Application guidelines for biosimilar medicines (European Medicines Agency and Food and Drug Administration)

Title Reference into effect

(A) General guidelines

(i) General characteristics

Guideline on similar biological medicinal products.

Scientific considerations in demonstrating biosimilarity to a reference product: guidance for industry.

(ii) Quality

Guideline on similar biological medicinal products containing biotechnology-derived proteins as active substance: quality issues.

(iii) Clinical and non-clinical trials

Guideline on comparability of biotechnology-derived medicinal products after a change in the manufacturing process. Non-clinical and clinical issues.

Guidelines on similar biological medicinal products containing biotechnology-derived proteins as active substance: non-clinical and clinical issues.

Clinical pharmacology data to support the demonstration of biosimilarity to a reference product: guidance for industry.

(iv) Immunogenicity

Guideline on immunogenicity assessment of therapeutic proteins.

Guideline on immunogenicity assessment of monoclonal antibodies intended for in vivo clinical use.

(v) Interchangeability

Considerations in demonstrating interchangeability with a reference product: guidance for industry.

(vi) Labelling

Labelling for biosimilar products: guidance for industry.

CHMP/437/04 Rev. 1

30 April 2015

1 April 2015

EMA/CHMP/BWP/247713/2012

1 December 2014

EMEA/CHMP/BMWP/101695/2006

1 November 2007

EMEA/CHMP/BMWP/42832/2005 Rev. 1

1 July 2015

1 December 2016

EMEA/CHMP/BMWP/14327/2006 Rev. 1

1 December 2017

EMA/CHMP/BMWP/86289/2010

1 December 2012

1 May 2019

1 July 2018

EMEA/CHMP/BMWP/301636/2008 Rev. 1

1 January 2019

Guideline on non-clinical and clinical development of similar biological medicinal products containing recombinant erythropoietins'.

Annex to guidelines on similar biological medicinal products containing biotechnology-derived proteins as active substance: non-clinical and clinical issues. Guidance on similar medicinal products containing recombinant granulocyte-colony stimulating factor (G-CSF).

Guideline on non-clinical and clinical development of similar biological medicinal products containing recombinant human insulin and insulin analogues.

Annex to guideline on similar biological medicinal products containing biotechnology-derived proteins as active substance: non-clinical and clinical issues. Guidelines on similar medicinal products containing somatropin.

Guideline on non-clinical and clinical development of similar biological medicinal products containing low-molecular-weight heparins

Guideline on non-clinical and clinical development of similar

biological medicinal products containing recombinant human follicle stimulating hormone (r-HFSH).

Guideline on similar biological medicinal products containing monoclonal antibodies - non-clinical and clinical issues.

Guideline on similar biological medicinal products containing interferon beta.
EMEA/CHMP/BMWP/31329/2005

1 June 2006

EMA/CHMP/BMWP/32775/2005 Rev. 1

1 September 2015

EMEA/CHMP/BMWP/94528/2005 Rev. 1 1 January 2019

EMEA/CHMP/BMWP/118264/2007 Rev. 1

EMA/CHMP/BMWP/671292/2010

1 June 2017

1 September 2013

EMA/CHMP/BMWP/403543/2010

1 December 2012

EMA/CHMP/BMWP/652000/2010

1 September 2013 justification of the relevance of comparative studies with the selected non-authorized comparator. Thus, in some cases, a bridging study might be necessary.

Due to the existing variability between the different batches of innovative medicine, regardless of the changes made during the fabrication process, the laboratory inter- ested in marketing a biosimilar medicine must guarantee the use of a representative and enough number of batches of the reference medicine for its proper characterization $^{8,31}$. Probably, one of the most significant challenges is the selection of the proper number of batches to handle the variability between the reference medicine and that 


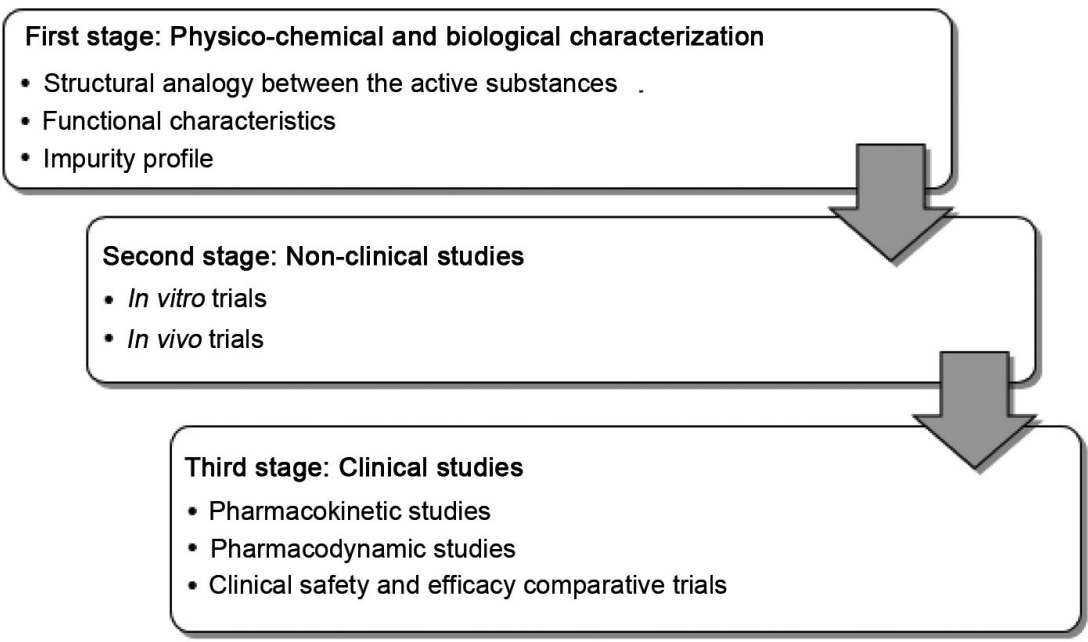

Figure 1. Stages in the development of biosimilar medicines (own creation).

for which the biosimilar condition is claimed. It seems clear that greater the number of batches of the reference medicine, more solid would be the development processes of biosimilar medicines, and therefore, this is the most convenient situation. So far, the EMA has not established any recommendations. The FDA, in a guidance that is awaiting approval, recommends the use of at least ten batches to characterize the reference medicine ${ }^{33}$.

\section{Requirements for biosimilar medicines}

The active substance of the biosimilar medicine must be as similar as possible to the active substance of the reference medicine, both in biological and molecular terms. For example, in the case of recombinant proteins, the amino acid sequence (primary structure) needs to be iden$\mathrm{tical}^{8,31}$. In some cases, it has been possible to mimic the secondary structure ${ }^{34}$, although this requirement is not essential to demonstrate biosimilarity.

Some differences are allowed between the reference biological medicine and the biosimilar medicine, in particular in the following aspects: dose (potency), pharmaceutical form, formulation, expression systems ${ }^{35}$ and administration system. However, it must be proved that the changes made by the applicant do not compromise the efficacy, safety or quality of the biosimilar medicine.

Once a biosimilar medicine has been authorized, the biosimilarity relationship will remain clear indefinitely. That is, in cases where changes in the reference or biosimilar medicine occur during the production process, the biosimilarity demonstration between two medicines through an extensive battery of studies would not be necessary, but the procedures applicable by each health authority in the industrial manufacturing processes of the medicines shall be adopted to evaluate the changes. The approach of this evaluation is based on the general principles established by the note for guidance on bio- technological/biological products subject to changes in their manufacturing process: comparability of biotechnological/biological products (CPMP/ICH/5721/03) (ICH Q5E).

However, biosimilar medicines are subject to surveillance during the post-authorization period, in order to continually control the benefit/risk balance, as well as to detect rare and uncommon adverse reactions that were not detected during the period prior to obtaining the marketing authorization. The applicable requirements in terms of pharmacovigilance for biosimilar medicines are similar to the ones established for the rest of the biological medicines ${ }^{24,36}$.

Although the regulatory authorities of the EU and USA do not demand to monitor the quality by design (QBD) principles for the development of biosimilar medicines, their compliance may present significant economic advantages for the applicant, as they allow to reduce both the time to market ${ }^{37}$, and the costs arising from the medicine manufacturing process ${ }^{38}$. Furthermore, we understand that complying with the QBD principles would also allow to improve the quality of the industrial manufacturing processes, which is translated in better quality guarantees for the medicine, and therefore, favourable for the patients.

\section{Principles to establish biosimilarity: comparability exercise}

Both the biosimilar medicine and the reference medicine must be highly similar in physico-chemical and biological terms $^{8,31}$. Any difference between them must not have significant implications in the efficacy or safety profile of the biosimilar medicine. The differences between the reference medicine and biosimilar medicine must be as restrictive as possible ${ }^{26}$.

During the development of biosimilar medicines, it is recommended to follow a comparative and stepwise 
approach. This process has three stages (Figure 1). The first stage consists of the full biological and physicochemical characterization of the biosimilar medicine, identifying the quality attributes as well as performing functional studies.

Depending on the strength of the data obtained during the first stage, as well as the mechanism of action of the active substances in the different pathologies where it is therapeutically useful, and the immunogenicity associated to the reference medicine, the reach and nature of the preclinical or non-clinical studies must be determined. Generally, it is not necessary to carry out carcinogenicity, teratogenicity or safety pharmacological trials. Besides, the EMA generally does not require carrying out local tolerance trials either.

Finally, it would be possible to start studies on human species. It is recommended to start with PK and PD trials, with adequate relevance and sensitivity ${ }^{31,32,39}$. The applicant must scientifically justify the selection of the group of subjects, the parameters to be evaluated, the dosage to trial, as well as the number of doses applied, etc.

Depending on the uncertainties detected in the PK/PD trials, the applicant must decide on the nature and extension of the clinical trials in order to demonstrate the biosimilarity (for example, clinical trials in stage I or stage III).

If the applicant chooses to conduct clinical trials aiming to eliminate the uncertainties obtained in previous studies to demonstrate biosimilarity, it is recommended to analyse the most sensitive therapeutic indication authorized for the reference medicine, and not the most relevant therapeutic indication. Therefore, it is not necessary to conduct clinical trials to confirm the clinical efficacy and safety in all the therapeutic indications claimed for the biosimilar medicine. Instead, both the EMA and FDA would allow an extrapolation of the indications, based on the whole evidence, after the individualized evaluation of every application, and in accordance with the updated scientific criteria ${ }^{8,31}$.

The extrapolation of indications is closely related to the concept of biosimilar medicine; hence its integration within the dimension of biosimilar medicines ${ }^{40}$. This relationship is evident from the fact that, to date, all biosimilar medicines authorized by the EMA and FDA have the same therapeutic indications approved with respect to the reference biological medicine.

This entire set of comparative studies between the reference medicine and the biosimilar medicine candidate will be evaluated by the regulatory authorities. This evaluation is known as the 'comparability exercise', which will be based on the whole evidence provided by the applicant $^{40}$.

The scientific principles governing the comparability exercise are equivalent to those applicable to the evaluation of the changes in the production process ${ }^{41}$. However, the comparability exercise required to authorize a biosi- milar medicine is much more comprehensive than the one to evaluate a change in the medicine manufacturing process, whether biological or not.

Ultimately, the importance of scientific evidence for the marketing authorization of biosimilar medicines is opposite to that of the reference medicines. For the latter, the weight of evidence is mainly found in clinical trials. However, in the case of biosimilar medicines, we look for comparative tests with high sensitivity that are able to detect differences between the two medicines ${ }^{42}$. Thus, the most relevant trials for biosimilar medicines are those included in their first stage of development (physicochemical and functional characterization).

Pharmacokinetic tests are also more sensitive detecting differences between two biological medicines with respect to clinical trials ${ }^{43}$.

Specific issues of the EMA: In relation to preclinical trials, local tolerance trials are not generally required. Regarding clinical data, initially the EMA did not allow the possibility to disregard clinical trials confirming the efficacy and safety of the biosimilar medicine ${ }^{30}$. This is probably one of the reasons why it has been criticized as being a particularly conservative and cautious institution $^{43}$. However, at present, clinical trials are not systematically required to grant marketing authorization for a given biosimilar medicine. To this end, the applicant must reliably demonstrate the biosimilarity relationship through quality-level studies, non-clinical studies, and where appropriate, through comparative PK/PD stu$\operatorname{dies}^{8,31}$. Nevertheless, the EMA does not allow the use of clinical data to justify the existence of significant differences in the quality attributes between a given reference medicine and a biosimilar medicine ${ }^{8}$.

For certain active substances (for example, granulocyte-colony stimulating factor, insulin), the EMA considers that the use of surrogate markers of clinical efficacy in PD trials is more appropriate than to conduct clinical re$\operatorname{search}^{44,45}$. Likewise, for low-molecular-weight heparins, the above-mentioned, the EMA does not consider it necessary to conduct clinical research during the developing process of biosimilar medicines ${ }^{46}$. For other active substances (teriparatide), no efficacy and safety comparative clinical trials are required for the authorization of the biosimilar medicine ${ }^{47}$.

\section{Specificities in the regulation of biosimilar medicines}

\section{Application scope}

Some medicines regulated as biological by the EMA (for example, those containing active substances like lowmolecular-weight heparins, etc.) have not been regulated by the FDA as such. This explains, in part, the differences 
in the regulation period of biosimilar medicines in the two regions.

\section{Identification guarantees}

EMA: In the EU, biosimilar medicines are identified through the international nonproprietary names (INNs), proposed by the World Health Organization (WHO), Geneva. This system gives each medicine a name according to the molecular structure and mechanism of action of the active substances. The first list of INNs was published in 1953.

While for some authors, INNs are appropriate for the identification of biosimilar medicines ${ }^{48}$, others call for a specific identification with the aim to carry out the prescription and dispensation processes with enough guarantees, as well as for the maintenance of clinical record data and to facilitate the pharmacovigilance tasks ${ }^{4}$.

FDA: In this context, two proposals to improve the identification of biosimilar medicines, which are similar have been put forward. One of them was facilitated by $\mathrm{WHO}^{49}$, but it was ruled out because of the lack of support by different stakeholders. The other proposal has been adopted by the FDA ${ }^{50}$.

In USA, biosimilar medicines are designated with a core name-equivalent to the $\mathrm{INN}$ - that would be hyphenated with a suffix formed by four small letters. The suffix would be proposed by the manufacturer, but would ultimately be granted by the FDA according to the defined criteria.

The purpose of adding the suffix is to differentiate the original biological medicine from the biosimilar medicines. This would reduce the possibility of making mistakes when prescribing and dispensing medicines, and thus also the likelihood of automatic substitution. On the other hand, the pharmacovigilance tasks would also be facilitated, since the suffix would identify the manufacturing laboratory of the biological medicine and would make it easier to target corrective measures only to the products concerned.

\section{Interchangeability}

EMA: The EMA does not require to test the interchangeability of medicines. Therefore, the EU member states are responsible for legislating on their territory on the interchangeability of biological medicines ${ }^{8}$.

FDA: On the other hand, the Biologics Price Competition and Innovation Act of 2009 distinguishes two types of biosimilar medicines, according to their potential interchangeability. Thus, not all biosimilar medicines are interchangeable in USA. In order for a medicine, that is similar to a reference medicine, to be considered interchangeable by the FDA, additional studies are required to demonstrate that the medicine produces the same clinical effect in patients as the reference medicine (section 351(k)(4)(A) Public Health Service Act).

If the applicant demonstrates that the biosimilar medicine is interchangeable with the reference medicine, then the FDA would grant one year of commercial exclusivity for the first biosimilar medicine marketed with a given active substance. In addition, the 'interchangeable' status would allow the biosimilar medicine to have a greater market share. These reasons undoubtedly justify interest of the pharmaceutical industry in carrying out interchangeability trials.

The extent of the data to demonstrate interchangeability of a biosimilar medicine with respect to the reference medicine is closely related to the active substance, and relates to the following aspects ${ }^{51}$ : (a) analysis of the critical quality attributes of the biosimilar medicine; (b) identification of analytical differences between the reference medicine and the proposed biosimilar medicine candidate; (c) analysis of the mechanism(s) in the therapeutic indications claimed, which in turn will be conditioned by: (i) the receptor(s) responsible for the pharmacological action, (ii) response to the dose or concentration of the medicine, (iii) structure-activity relationship of the medicine, (iv) location and expression of the receptor(s) involved in the pharmacological action responsible for the therapeutic effect and (d) analysis of the differences related to: (i) pharmacokinetics, (ii) immunogenicity, (iii) expected toxicity under usual conditions of use between the reference medicine and the biosimilar medicine.

For demonstration of the therapeutic equivalence between the innovative medicine and the biosimilar medicine, the FDA has established the following requirements $^{51}$ : (a) the form of administration studied must be the most sensitive; (b) the most sensitive authorized therapeutic indication must be evaluated; (c) the reference medicine must have been authorized by the FDA. If the applicant decides to conduct the trial with healthy individuals, to evaluate another form of administration, or to use a purchaser not authorized in USA, he/she must provide scientific justification for any of these facts, in order for the FDA to admit completion of the studies.

The interchangeability of the two medicines can be demonstrated through an independent study, or it can be determined through efficacy and safety confirmatory clinical trials ${ }^{52}$. In any case, in order for the biosimilar medicine to be considered interchangeable with the reference medicine, no significant differences in PK and PD parameters, or in the safety profile (adverse effects, immunogenicity, etc.) shall be admitted.

\section{Proposals for the modification of biosimilarity requirements}

The scientific community has suggested reducing the regulatory requirements to obtain a marketing authorization 
for biosimilar medicines in the EU, given the experience gained with such medicines ${ }^{53}$. This fact would be an incentive for the pharmaceutical industry, since it would reduce the research and development costs for biosimilar medicines, and would allow them to be authorized promptly and, ultimately would result in a series of health and economic benefits for all stakeholders.

Cohen et al. ${ }^{54}$ mention that improvements in the knowledge of biological medicines, together with the advances in science, will allow the reliable demonstration of biosimilarity through analytical techniques. Agbogbo et al. ${ }^{37}$ propose the use of protein dynamics techniques in order to study the possible interactions of the biosimilar medicine, to obtain a deeper analytical knowledge of it, as well as of the structure-activity relationship and, ultimately to strengthen the demonstration of the relationship of biosimilarity with the reference medicine ${ }^{37}$.

Velasco-Velázquez et al. ${ }^{55}$ have shown that in the case of medicines containing infliximab as an active substance, pre-clinical research is enough to elucidate the uncertainties generated regarding the structural and functional similarity of the reference biological medicine and the biosimilar medicine.

Although in many cases, the EMA recommends to conduct clinical trials in phase III to demonstrate the biosimilarity relationship for medicines containing certain active substances (for example, erythropoietin, granulocytecolony stimulating factor, somatropin, interferon beta, recombinant human follicle stimulating hormone, monoclonal antibodies $)^{44,56-60}$, Frapaise $^{61}$ concludes that they provide information of little value for the demonstration of biosimilarity. He proposes to clear up the residual uncertainties of the analytical and pre-clinical studies through clinical trials in phase I, in order to reduce the developing costs of biosimilar medicines, and ultimately to facilitate their access to patients ${ }^{61}$. Nevertheless, many clinical trials in phase III have been conducted so far to confirm the similarity of clinical safety and efficacy of biosimilar medicines ${ }^{62-64}$.

Wolff-Holz et al $^{43}$ consider that in certain cases (for example, for biosimilar medicines with simpler structures), clinical safety and efficacy comparative trials (in phase I and/or III) could be replaced by PK and PD studies ${ }^{43}$.

If the clinical trials were necessary to demonstrate biosimilarity, in order to reduce the sample size needed, Clark et al. ${ }^{65}$, recommend the use of a 'synthetic' design. This kind of design would have been appropriate, for example, for certain biosimilar medicines.

Webster et al. ${ }^{66}$ proposed an alternative model to evaluate biosimilarity. According to them, to demonstrate the biosimilarity relationship between two medicines, it would be enough to obtain solid evidence at the analytical and pharmacokinetic levels. Therefore, they proposed to replace the evaluation of all the evidence for a more efficient model, which they called 'confirmation of sufficient likeness ${ }^{\prime 66}$. This model would avoid the execution of efficacy and safety comparative clinical trials, in vivo pre-clinical studies, etc.

\section{Conclusion}

The EMA and FDA have developed frameworks to regulate biosimilar medicines. These medicines are developed through a marketing authorization accelerated procedure, based on the demonstration of similarity with a reference medicine that has been previously authorized by the competent health authorities, which could be marketed once the legal elements preventing their market entry (patent and health data protection period of the reference medicine) have expired.

Although the basic principles to demonstrate biosimilarity are the same among the different regulatory agencies, there are significant differences in certain aspects (application scope, identification guarantees and interchangeability).

Due to the experience acquired with biosimilar medicines as well as with scientific advances, the latest recommendations published by the EMA have seen a decrease in the requirements for the demonstration of biosimilarity through clinical trials. This approach has also been subsequently adopted by the FDA. We consider that the recent regulatory changes applied are appropriate, insofar as they allow the early marketing of biosimilar medicines, without undermining the health guarantees required for them and ultimately, facilitating their accessibility to patients.

Several studies have proposed greater emphasis on the demonstration of biosimilarity through physico-chemical, functional and non-clinical studies ${ }^{43,55,66}$. In other words, clinical research is becoming less important in demonstrating biosimilarity. In fact, it is expected that biosimilar medicines may be authorized without having to conduct clinical trials.

1. EMA, Procedural advice for users of the centralised procedure for similar biological medicinal products applications, European Medicines Agency, EMA/940451/2011, 2012; https://www.ema. europa.eu/en/documents/regulatory-procedural-guideline/europeanmedicines-agency-procedural-advice-users-centralised-proceduresimilar-biological-medicinal_en-0.pdf (accessed on 28 February 2020).

2. FDA, Biologics Price Competition and Innovation Act of 2009, Food and Drug Administration, 2009; https:/www.govinfo.gov/ content/pkg/FR-2010-12-08/pdf/2010-30713.pdf (accessed on 28 February 2020).

3. Kurki, P., van Aerts, L., Wolff-Holz, E., Giezen, T., Skibeli, V. and Weise, M., Interchangeability of biosimilars: a European perspective. BioDrugs, 2017, 31, 83-91.

4. Grampp, G. and Ramanan, S., The diversity of biosimilar design and development: implications for policies and stakeholders. BioDrugs, 2015, 29, 365-372.

5. de Mora, F., Biosimilar: what it is not. Br. J. Clin. Pharmacol., 2015, 80, 949-956. 
6. Meher, B. R., Balan, S., Mohanty, R. R., Jena, M. and Das, S., Biosimilars in India; current status and future perspectives. J. Pharm. Bioallied Sci., 2019, 11, 12-15.

7. Saberwal, G., India to power the more widespread use for biosimilars? Curr. Sci., 2019, 116, 1451-1452.

8. EMA, Guideline on similar biological medicinal products, European Medicines Agency, CHMP/437/04 Rev 1, 23 October 2014; https://www.ema.europa.eu/en/documents/scientific-guideline/ guideline-similar-biological-medicinal-products-rev1_en.pdf (accessed on 28 February 2020).

9. FDA, Biosimilar Development, Review, and Approval, Food and Drug Administration, 2019; http://www.fda.gov/drugs/biosimilars/ biosimilar-development-review-and-approval (accessed on 28 February 2020).

10. Van de Vooren, K., Curto, A. and Garattini, L., Biosimilar versus generic drugs: same but different? Appl. Health Econ. Health Policy, 2015, 13, 125-127.

11. Duivelshof, B. L., Jiskoot, W., Beck, A., Veuthey, J.-L., Guillarme, D. and D'Atri, V., Glycosylation of biosimilars: recent advances in analytical characterization and clinical implications. Anal. Chim. Acta, 2019, 1089, 1-18.

12. Xiao, Z. et al., A comprehensive approach for evaluating charge heterogeneity in biosimilars. Eur. J. Pharm. Sci., 2018, 115, 1924.

13. Delgado Sánchez, O., Ginard Vicens, D., Sampol Mayol, A. and Terrasa Pons, J., Biosimilar medicines: impact, opportunities and strategies, Twelve years of experience in Europe. Med. Clín., 2019, 152, 411-415.

14. Girault, D. et al., Biosimilars: from technical to pharmacoeconomic considerations. Thérapie, 2015, 70, 47-55.

15. Dutta, A. S., Huys, I., Vulto, A. G., and Simoens, S., Identifying key benefits in European off-patent biologics and biosimilars markets: it is not only about price! BioDrugs, 2019, 34, 203-211.

16. Cuellar, S., McBride, A. and Medina, P., Pharmacist perspectives and considerations for implementation of therapeutic oncology biosimilars in practice. Am. J. Health Syst. Pharm., 2019, 76, 1725-1738.

17. Bellinvia, S., Cummings, J. R. F., Ardern-Jones, M. R. and Edwards, C. J., Adalimumab biosimilars in Europe: an overview of the clinical evidence. BioDrugs, 2019, 33, 241-253.

18. Haustein, R., de Millas, C., Höer, A. and Häussler, B., Saving money in the European healthcare systems with biosimilars. Generics Biosimilars Initiat. J., 2012, 1, 120-126.

19. Kim, D., Transparency policies of the European medicines agency: has the paradigm shifted. Med. Law Rev., 2017, 25, 456-483.

20. Noguera Peña, A., La polémica en torno a la sustitución e intercambiabilidad de los medicamentos biosimilares en el ordenamiento jurídico español. Derecho Salud, 2019, 29, 5-52.

21. González, A. et al., La introducción de los biosimilares en España. Estimación del ahorro para el Sistema Nacional de Salud, Fundación Weber, Madrid, 2017.

22. Gascon, P., Krendyukov, A., Mathieson, N., Natek, M. and Aapro, M., Extrapolation in practice: lessons from 10 years with biosimilar filgrastim. BioDrugs, 2019, 33, 635-645.

23. Civoli, F. et al., Recommendations for the development and validation of immunogenicity assays in support of biosimilar programs. AAPS J., 2020, 22, 1-9.

24. Zúñiga Hernando, L. and Calvo Hernáez, B., Biosimilars: pharmacovigilance and risk management. Pharmacoepidemiol. Drug Saf., 2010, 19, 661-669.

25. Łukasik, Z. and Nowicki, M., Knowledge and attitude of community pharmacy employees towards an automatic drug substitution of generics and biosimilars. Acta Pol. Pharm., 2018, 75, 12471254 .

26. Tsiftsoglou, A. S., Ruiz, S. and Schneider, C. K., Development and regulation of biosimilars: current status and future challenges. BioDrugs, 2013, 27, 203-211.
27. McKinnon, R. A. et al., Biosimilarity and interchangeability: principles and evidence: a systematic review. BioDrugs, 2018, 32, $27-$ 52.

28. Haghnejad, V., Le Berre, C., Dominique, Y., Zallot, C., Guillemin, F. and Peyrin-Biroulet, L., Impact of a medical interview on the decision to switch from originator infliximab to its biosimilar in patients with inflammatory bowel disease. Dig. Liver Dis., 2020, 52, 281-288.

29. Cohen, H. P., Blauvelt, A., Rifkin, R. M., Danese, S., Gokhale, S. B. and Woollett, G., Switching reference medicines to biosimilars: a systematic literature review of clinical outcomes. Drugs, 2018, 78, 463-478.

30. EMA, Guideline on similar biological medicinal products, European Medicines Agency, CHMP/437/04, 30 October 2005; https://www.ema.europa.eu/documents/scientific-guideline/guideline-similar-biological-medicinal-products-first-version_en.pdf (accessed on 28 February 2020).

31. FDA, Scientific considerations in demonstrating biosimilarity to a reference product, Food and Drug Administration, 2015; https://www.fda.gov/media/82647/download (accessed on 28 February 2020).

32. EMA, Guideline on similar biological medicinal products containing biotechnology-derived proteins as active substance: nonclinical and clinical issues, European Medicines Agency, EMEA/ CHMP/BMWP/42832/2005 Rev. 1, 2014; https://www.ema. europa.eu/en/documents/scientific-guideline/guideline-similarbiological-medicinal-products-containing-biotechnology-derivedproteins-active_en-2.pdf (accessed on 28 February 2020).

33. FDA, Development of therapeutic protein biosimilars: comparative analytical assessment and other quality-related considerations: guidance for industry, Food and Drug Administration, 2018; https://www.fda.gov/media/125484/download (accessed on 28 February 2020).

34. Jung, S. K. et al., Physicochemical characterization of Remsima ${ }^{\circledR}$. $m A b s, 2014,6,1163-1177$.

35. Dent, M. and Matoba, N., Cancer biologics made in plants. Curr. Opin. Biotechnol., 2020, 61, 82-88.

36. Singh, A., Kalaivani, M., Srivastava, S., Goyal, R. K. and Gupta, S. K., Postmarketing safety of biosimilars: current status, challenges, and opportunities in the spontaneous reporting system. Ther. Innov. Regul. Sci., 2019.

37. Agbogbo, F. K. et al., Current perspectives on biosimilars. J. Ind. Microbiol. Biotechnol., 2019, 46, 1297-1311.

38. Vessely, C. and Bussineau, C., QbD in biopharmaceutical manufacturing and biosimilar development. In Biosimilars: Regulatory, Clinical, and Biopharmaceutical Development (eds Gutka, H., Yang, H. and Kakar, S.), Springer Healthcare, Cham, 2018, pp. 187-220.

39. FDA, Clinical pharmacology data to support a demonstration of biosimilarity to a reference product, Food and Drug Administration, 2016; https://www.fda.gov/media/88622/download (accessed on 28 February 2020).

40. Curigliano, G., O’Connor, D. P., Rosenberg, J. A. and Jacobs, I., Biosimilars: extrapolation for oncology. Crit. Rev. Oncol. Hematol., 2016, 104, 131-137.

41. Vezér, B., Buzás, Z., Sebeszta, M. and Zrubka, Z., Authorized manufacturing changes for therapeutic monoclonal antibodies (mAbs) in European Public Assessment Report (EPAR) documents. Curr. Med. Res. Opin., 2016, 32, 829-834.

42. Chamberlain, P. and Kurki, P., Immunogenicity assessment of biosimilars: a multidisciplinary perspective. In Biosimilars: Regulatory, Clinical, and Biopharmaceutical Development (eds Gutka, H., Yang, H. and Kakar, S.), Springer Healthcare, Cham, Switzerland, 2018, pp. 489-542.

43. Wolff-Holz, E., Tiitso, K., Vleminckx, C. and Weise, M., Evolution of the EU biosimilar framework: past and future. BioDrugs, $2019,33,621-634$. 
44. EMEA, Annex to guideline on similar biological medicinal products containing biotechnology-derived proteins as active substance: non-clinical and clinical issues. Guidance on similar medicinal products containing recombinant granulocyte-colony stimulating factor (G-CSF), European Medicines Evaluation Agency, EMEA/CHMP/BMWP/31329/2005, 22 February 2006; https://www.ema.europa.eu/documents/scientific-guideline/annexguideline-similar-biological-medicinal-products-containing-biotechnology-derived-proteins_en.pdf (accessed on 28 February 2020).

45. EMA, Guideline on non-clinical and clinical development of similar biological medicinal products containing recombinant human insulin and insulin analogues, European Medicines Agency, EMA/CHMP/BMWP/32775/2005_Rev. 1, 26 February 2015; https://www.ema.europa.eu/documents/scientific-guideline/guidelinenon-clinical-clinical-development-similar-biological-medicinalproducts-containing_en-0.pdf (accessed on 28 February 2020).

46. European Medicines Agency, Guideline on non-clinical and clinical development of similar biological medicinal products containing low-molecular-weight heparins (EMEA/CHMP/BMWP/ 118264/2007 Rev. 1). 20 November 2016; https://www.ema. europa.eu/documents/scientific-guideline/guideline-non-clinicalclinical-development-similar-biological-medicinal-productscontaining-low_en.pdf (accessed on 28 February 2020).

47. Kovács, P. et al., Comparative physicochemical and biological characterization of the similar biological medicinal product teriparatide and its reference medicinal product. BioDrugs, 2020, 34, $65-75$.

48. Genazzani, A. A. et al., Biosimilar drugs: concerns and opportunities. BioDrugs, 2007, 21, 351-356.

49. WHO, Biological Qualifier. An INN Proposal, World Health Organization, July 2014; http://www.who.int/medicines/services/ inn/bq_innproposal201407.pdf?ua=1 (accessed on 28 February 2020).

50. FDA, Nonproprietary naming of biological products guidance for industry, Food and Drug Administration, 2017; http://www.fda. gov/downloads/drugs/guidancecomplianceregulatoryinformation/ guidances/ucm459987.pdf (accessed on 28 February 2020).

51. FDA, Considerations in demonstrating interchangeability with a reference product guidance for industry, Food and Drug Administration, 2019; https://www.fda.gov/media/124907/download (accessed on 28 February 2020).

52. Chance, K., FDA expectations for demonstrating interchangeability. Ther. Innov. Regul. Sci., 2018, 52, 369-373.

53. Vulto, A. G., Delivering on the promise of biosimilars. BioDrugs, 2019, 33, 599-602.

54. Cohen, H. P., Lamanna, W. C. and Schiestl, M., Totality of evidence and the role of clinical studies in establishing biosimilarity. In Biosimilars: Regulatory, Clinical and Biopharmaceutical Development (eds Gutka, H., Yang, H. and Kakar, S.), Springer Healthcare, Cham, Switzerland, 2018, pp. 601-628.

55. Velasco-Velázquez, Ma. et al., Extensive preclinical evaluation of an infliximab biosimilar candidate. Eur. J. Pharm. Sci., 2017, 102, $35-45$.

56. EMEA, Annex to guideline on similar biological medicinal products containing biotechnology-derived proteins as active substance: non-clinical and clinical issues, Guideline on similar medicinal products containing somatropin, European Medicines Evaluation Agency. EMEA/CHMP/BMWP/94528/2005 Rev. 1, 28
June 2018; https://www.ema.europa.eu/documents/scientificguideline/annex-guideline-similar-biological-medicinal-productscontaining-biotechnology-derived-proteins_en-1.pdf (accessed on 28 February 2020).

57. EMA, Guideline on similar biological medicinal products containing interferon beta, European Medicines Agency, EMA/CHMP/ BMWP/652000/2010, 21 February 2013; https://www.ema. europa.eu/documents/scientific-guideline/guideline-similar-biological-medicinal-products-containing-interferon-beta_en.pdf (accessed on 28 February 2020).

58. EMA, Guideline on nonclinical and clinical development of similar biological medicinal products containing recombinant erythropoietins (Revision), European Medicines Agency, EMEA/CHMP/ BMWP/301636/2008 Rev. 1, 23 July 2018; https://www.ema. europa.eu/en/documents/scientific-guideline/guideline-non-clinical-clinical-development-similar-biological-medicinal-productscontaining_en-1.pdf (accessed on 28 February 2020).

59. EMA, Guideline on non-clinical and clinical development of similar biological medicinal products containing recombinant human follicle stimulating hormone ( $\mathrm{r}-\mathrm{hFSH})$, European Medicines Agency, EMA/CHMP/BMWP/671292/2010), 21 February 2013; https://www.ema.europa.eu/documents/scientific-guideline/guideline-similar-biological-medicinal-products-containing-interferonbeta_en.pdf (accessed on 28 February 2020).

60. EMA, Guideline on similar biological medicinal products containing monoclonal antibodies - non-clinical and clinical issues, European Medicines Agency, EMA/CHMP/BMWP/403543/2010, 30 May 2012; https://www.ema.europa.eu/en/documents/scientific-guideline/guideline-similar-biological-medicinal-productscontaining-monoclonal-antibodies-non-clinical_en.pdf (accessed on 28 February 2020).

61. Frapaise, F.-X., The end of phase 3 clinical trials in biosimilars development? BioDrugs, 2018, 32, 319-324.

62. Ye, B. et al., Efficacy and safety of biosimilar CT-P13 compared with originator infliximab in patients with active Crohn's disease: an international, randomised, double-blind, phase 3 non-inferiority study. Lancet, 2019, 393, 1699-1707.

63. Jaworski, J. et al., Switch from reference etanercept to SDZ ETN, an etanercept biosimilar, does not impact efficacy, safety, and immunogenicity of etanercept in patients with moderate-to-severe rheumatoid arthritis: 48-week results from the phase III, randomized, double-blind EQUIRA study. Arthritis Res. Ther., 2019, 21.

64. Kim, Y. et al., Efficacy and safety of CKD-11101 (proposed biosimilar of darbepoetin-alfa) compared with darbepoetin-alfa in patients on hemodialysis: a randomized, double-blinded, parallelgroup phase III study. BioDrugs, 2020, 34, 99-110.

65. Clark, T., Jo, S. J. and Phillips, A., Sample size for biosimilar trials: in defense of synthesis. Ther. Innov. Regul. Sci., 2018, 52, 300-305.

66. Webster, C. J., Wong, A. C. and Woollett, G. R., An efficient development paradigm for biosimilars. BioDrugs, 2019, 33, 603611.

Received 28 February 2020; revised accepted 20 September 2020

doi: $10.18520 / \mathrm{cs} / \mathrm{v} 120 / \mathrm{i} 1 / 56-65$ 